\title{
Universiteit
}

Leiden

The Netherlands

\section{Magnetic excitations in infinite-layer nickelates}

Lu, H.; Rossi, M.; Nag, A.; Osada, M.; Li, D.F.; Lee, K.; ... ; Lee, W.S.

\section{Citation}

Lu, H., Rossi, M., Nag, A., Osada, M., Li, D. F., Lee, K., ... Lee, W. S. (2021). Magnetic excitations in infinite-layer nickelates. Science, 373(6551), 213-216. doi:10.1126/science.abd7726

Version: $\quad$ Publisher's Version

License: $\quad$ Licensed under Article 25fa Copyright Act/Law (Amendment Taverne)

Downloaded from: $\quad$ https://hdl.handle.net/1887/3248669

Note: To cite this publication please use the final published version (if applicable). 


\section{SUPERCONDUCTIVITY}

\section{Magnetic excitations in infinite-layer nickelates}

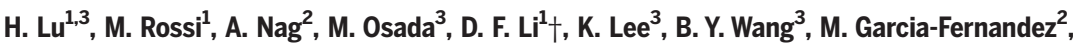 \\ S. Agrestini ${ }^{2}$, Z. X. Shen ${ }^{1,3}$, E. M. Been ${ }^{1}$, B. Moritz ${ }^{1}$, T. P. Devereaux ${ }^{1,3,4}$, J. Zaanen ${ }^{5}$, \\ H. Y. Hwang ${ }^{1,3}$, Ke-Jin Zhou ${ }^{2 *}$, W. S. Lee ${ }^{1 *}$
}

The discovery of superconductivity in infinite-layer nickelates brings us tantalizingly close to a material class that mirrors the cuprate superconductors. We measured the magnetic excitations in these nickelates using resonant inelastic x-ray scattering at the $\mathrm{Ni} L_{3}$-edge. Undoped $\mathrm{NdNiO}_{2}$ possesses a branch of dispersive excitations with a bandwidth of approximately 200 milli-electron volts, which is reminiscent of the spin wave of strongly coupled, antiferromagnetically aligned spins on a square lattice. The substantial damping of these modes indicates the importance of coupling to rare-earth itinerant electrons. Upon doping, the spectral weight and energy decrease slightly, whereas the modes become overdamped. Our results highlight the role of Mottness in infinite-layer nickelates.

T he mechanism of unconventional, hightemperature superconductivity, as embodied in families of copper oxides, or cuprates, remains a highly controversial subject in condensed matter physics. However, the mechanism is only one of a number of interrelated unresolved questions, which include the origins of the strange metal phase and the implications of intertwined orders and push the limits of established mathematical theory. Soon after the discovery of cuprates, the late P. W. Anderson recognized that this strangeness can be traced back to the strong local electron repulsion (Hubbard $U$ ) and the peculiar properties of doped Mott insulatorsMottness-which remains challenging to renormalize to more conventional Fermi-liquid or Bardeen-Cooper-Schrieffer (BCS) physics $(1,2)$. Although large-scale classical simulations of Hubbard-type models have acquired benchmarking status and will be used to validate the first generation of quantum computers (3), string-theoretical methods can help make the case that the strangeness of cuprate electrons originates in dense, many-body quantum entanglement (4).

Although many cuprate families exist, it has proven very hard to find other material classes, based on different transition metals, that exhibit similar Mott physics, let alone the quasitwo-dimensional structure and small quantum spins that are deemed to be essential. The land-

Stanford Institute for Materials and Energy Sciences, SLAC National Accelerator Laboratory and Stanford University, Menlo Park, CA 94025, USA. ²Diamond Light Source, Harwell Campus, Didcot OX11 ODE, UK. ${ }^{3}$ Geballe Laboratory for Advanced Materials, Departments of Physics and Applied Physics, Stanford University, Stanford, CA 94305, USA. ${ }^{4}$ Department of Materials Science and Engineering, Stanford University, Stanford, CA 94305, USA. ${ }^{5}$ nstituut-Lorentz for theoretical Physics, Leiden University, Niels Bohrweg 2, 2333 CA Leiden, Netherlands.

*Corresponding author. Email: kejin.zhou@diamond.ac.uk (K.-J.Z.); leews@stanford.edu (W.S.L.) †Present address: Department of Physics, City University of Hong Kong, Kowloon, Hong Kong, China.
A

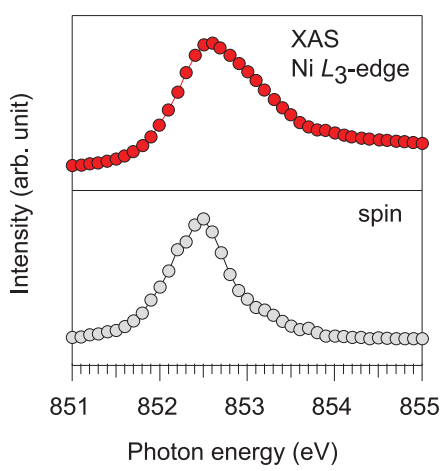
$(\sim 0.2 \mathrm{eV})$, and phonon $(\sim 0.07 \mathrm{eV})$, respectively. scape changed very recently with the discovery of superconductivity in doped monovalent infinite-layer nickelates $(5,6)$. Their crystal structures are very similar to the cuprates, with $\mathrm{NiO}_{2}$ planes separated by spacer layers that contain a minimal set of chemical elements, which is simpler than most of the cuprates. They were predicted (7-17) to be isoelectronic to the cuprates: monovalent $\mathrm{Ni}^{+}$characterized by the same $3 d^{9}$ state as that of $\mathrm{Cu}^{2+}$ in the cuprates.

Are the nickelates really cuprate cousins in the most important way, Mottness? There exists considerable uncertainty regarding the local Coulomb repulsion energy $U$ because the atomic $d$-orbitals of monovalent $\mathrm{Ni}^{+}$are more extended than those of divalent $\mathrm{Cu}^{2+}$, a factor that can have a large influence on the magnitude of $U$. The magnetic structure can provide a constraint that allows for proper categoriza- tion. In a weakly interacting metal, the magnetic excitations spread over the full bandwidth $(W$ $3 \mathrm{eV}$ ), whereas in a spin-density-wave-like system $(U \ll W)$, the excitations accumulate at low energies and exclusively near the ordering wave vectors. At higher energies (near the magnetic zone boundaries), these "paramagnons" should be completely overdamped, decaying in the metallic continuum (Landau damping). However, in a Mott insulator $(U \gtrsim W)$, the metallic continuum is "pushed up" by $U$, and instead one is dealing with the excitations of a pure spin system, which is characterized by long-lived propagating spin waves that survive all the way up to the zone boundary. Furthermore, information about magnetic excitations can clarify the debated energy scale of the spin exchange coupling strength $J$ in $\mathrm{NdNiO}_{2}$; some theories suggest $J$ to be one order of magnitude smaller than in cuprates owing to a large charge transfer energy (11-14), whereas some others argue differently (15-17).

To address these questions, we report here the measurements of the spin excitation spectra in infinite-layer nickelates by using resonant inelastic x-ray scattering (RIXS). We studied 10-nm-thick films of $\mathrm{Nd}_{1-x} \mathrm{Sr}_{x} \mathrm{NiO}_{2}$ grown on a $\mathrm{SrTiO}_{3}$ substrate with a $\mathrm{SrTiO}_{3}$ capping layer of a few unit cells to maintain and orient the nickelate crystalline structure (figs. S1 and S2) $(5,18)$. The Ni $L_{3}$-edge x-ray absorption (XAS) spectrum of $\mathrm{NdNiO}_{2}$ is shown in Fig. 1A, top, which exhibits a single peak that corresponds primarily to the $2 p^{6} 3 d^{9} \rightarrow 2 p^{5} 3 d^{10}$ transition (10), as in cuprates. Upon doping with $\mathrm{Sr}$, the XAS broadens (fig. S3), which is consistent with the recent report from an electron energy loss spectroscopy measurement with a scanning transmission electron microscope (19). For RIXS
B

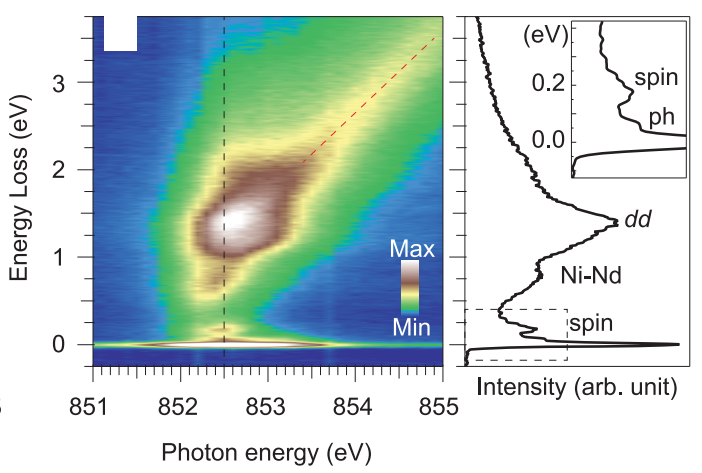

Fig. 1. XAS and RIXS map of elementary excitations in $\mathrm{NdNiO}_{2}$. (A) (Top) $\mathrm{Ni} L_{3}$-edge XAS measured with total electron yield at $20 \mathrm{~K}$ in normal incidence geometry. (Bottom) Resonant profile of spin-magnetic excitations obtained by integrating the RIXS intensity between 0.1 and $0.26 \mathrm{eV}$. (B) RIXS intensity map versus energy loss and incident photon energy across the Ni $L_{3}$-edge at $20 \mathrm{~K}$. (Right) RIXS spectrum taken at a photon energy of $852.5 \mathrm{eV}$ (black dashed line). The red dashed line indicates the fluorescence feature. (Inset) An enlarged view of the spectra within the dashed box. dd, Ni-Nd, spin, and ph denote orbital excitations within the $\mathrm{Ni} 3 d$ orbitals, spectral feature of $\mathrm{Ni} 3 d$ and $\mathrm{Nd} 5 d$ orbital hybridization, magnetic excitation 
measurements, we adopted an experimental geometry well established to measure magnetic spin excitations in transition-metal oxides (20-27), including cuprates. A hierarchy of excitations can be resolved in a RIXS intensity map acquired by tuning the incident photon energy across the Ni $L_{3}$-edge (Fig. $1 \mathrm{~B}$ ): a fluorescence feature at $3 \mathrm{eV}$ and above (Fig. 1B, red dashed line), dipole-forbidden $d d$-excitations from 1.2 to $3.0 \mathrm{eV}$, a peak at $\sim 0.7 \mathrm{eV}$ due to hybridization between $\mathrm{Ni} 3 d$ and Nd $5 d$ orbitals (10), and a low-energy feature at $\sim 0.2 \mathrm{eV}$ whose intensity is maximal near the peak of XAS (Fig. $1 \mathrm{~A}$, bottom) and is reminiscent of the magnetic excitations seen in RIXS maps of cuprates taken at the $\mathrm{Cu} \mathrm{L}_{3}$-edge (20-22). At lower energies, phonon excitations at $\sim 0.07 \mathrm{eV}$ can also be resolved (Fig. 1B).

To characterize the behavior of these lowenergy excitations, we measured detailed momentum-resolved RIXS maps (27). As shown in Fig. 2, A and B, the excitations bear a strong resemblance to spin-1/2 antiferromagnet (AFM) magnons on a square lattice. Namely, they disperse strongly with maxima at $(0.5,0)$ and $(0.25$, $0.25)$, soften toward the conventional AFM ordering wave vector $(0.5,0.5)$, and exhibit spectral intensity suppression near $(0.5,0)(27)$. The magnetic excitations do not exhibit obvious dispersion along the $c$ axis (Fig. 2C), indicating that they are quasi-two-dimensional. We fit the spectra to a damped harmonic oscillator (DHO) function $\chi^{\prime \prime}(q, \omega)$ (fig. S4) $(22,27)$, given by

$$
\chi^{\prime \prime}(q, \omega)=\frac{\gamma_{q} \omega}{\left(\omega^{2}-\varepsilon_{q}^{2}\right)^{2}+4 \gamma_{q}^{2} \omega^{2}}
$$

where $\varepsilon_{q}$ is the undamped mode energy and $\gamma_{q}$ is the damping factor. The DHO function is equivalent to an antisymmetrized Lorentzian function (21) in the under-damped condition $\left(\gamma_{q} \ll \varepsilon_{q}\right)$, but it provides a more physical description in strongly damped conditions (22). The fitted $\varepsilon_{q}$ and $\gamma_{q}$ along the three highsymmetry directions are shown in Fig. 3, except for the data near $(0,0)$, where the fitting is unreliable because the magnetic mode merges with the phonon and the tail of the elastic peak. The dispersion extracted from the DHO function is similar to that extracted by an antiLorentzian function, which essentially tracks the peak position of the spectrum (fig. S5). We also found a noticeable dispersion of $\sim 50 \mathrm{meV}$ along the AFM zone boundary [Figs. $2 \mathrm{~A}$ and $3 \mathrm{~A}$, the $(h, 0.5-h)$ direction], which is indicative of substantial exchange interactions beyond nearest-neighbor $\mathrm{Ni}$ (28). We fit the extracted dispersion to a linear spin wave form for the spin-1/2 square-lattice Heisenberg AFM (29), including nearest- and next-nearest-neighbor exchange couplings

$$
H=J_{1} \sum_{\langle i, j\rangle} S_{i} \cdot S_{j}+J_{2} \sum_{\left\langle i, i^{\prime}\right\rangle} S_{i} \cdot S_{i^{\prime}}
$$

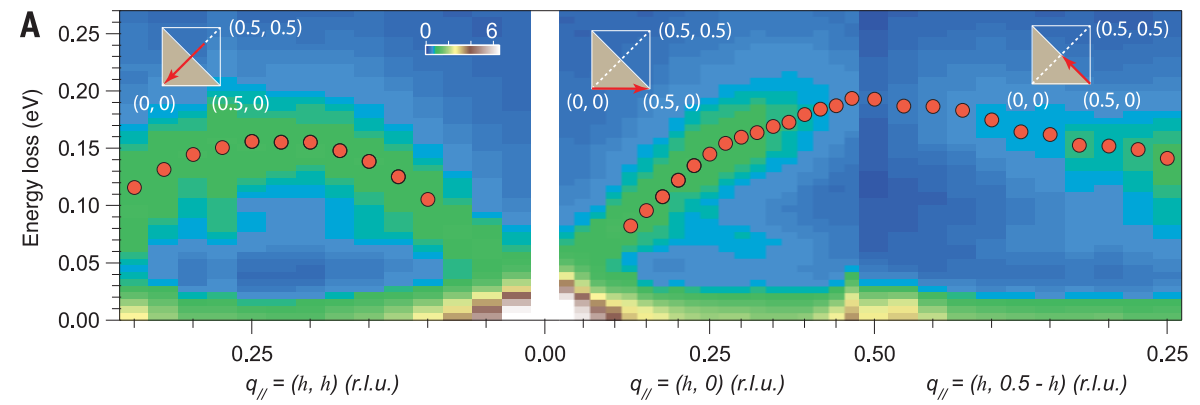

B
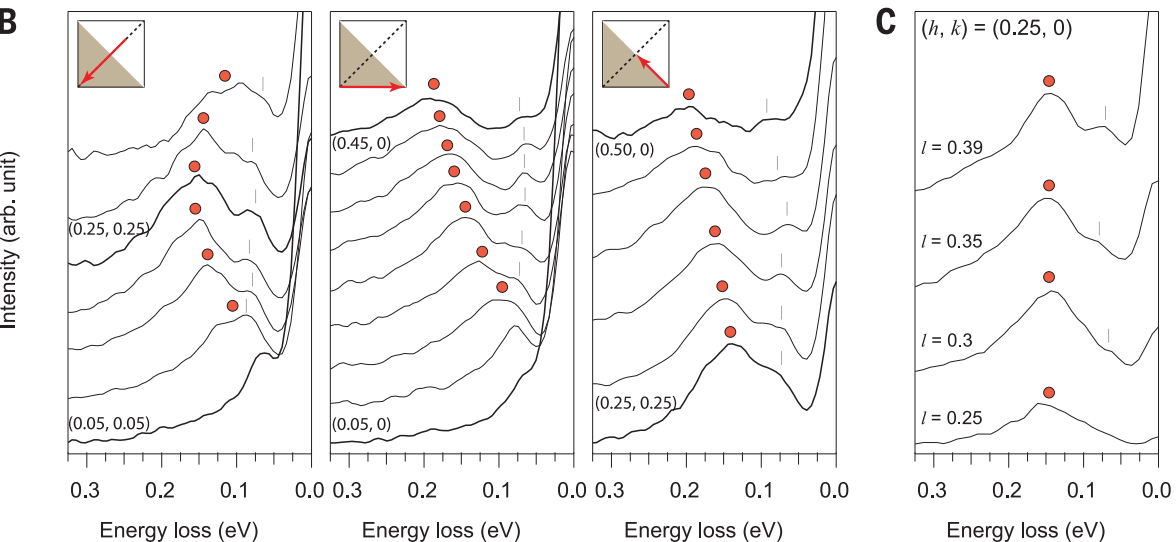

Fig. 2. Momentum-dependent RIXS intensity maps of $\mathbf{N d N i O}_{2}$. (A) RIXS intensity maps versus energy loss and projected in-plane momentum transfer along three high-symmetry directions, as indicated with red arrows in the insets, which show a Brillouin zone with the first AFM zone shaded. Measurements were taken at $20 \mathrm{~K}$. The red circles indicate peak positions of the magnetic excitation spectra. (B) Raw RIXS spectra at representative projected in-plane momentum transfers. The red circles indicate the peak positions of magnetic excitations, and the gray ticks indicate phonon excitations. (C) Raw RIXS spectra measured at a fixed in-plane momentum $(0.25,0)$ with different out-of-plane momentum $\mathbf{I}$.

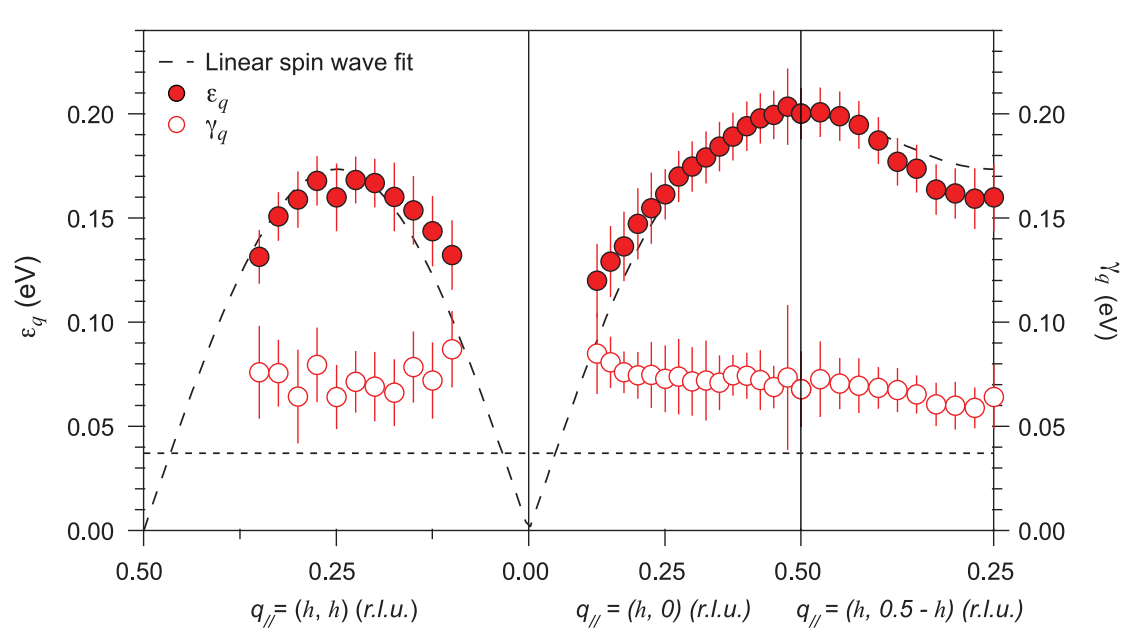

Fig. 3. Dispersion of magnetic excitations in $\mathrm{NdNiO}_{2}$ and fit to the linear spin wave theory. A summary of fitted magnetic mode energy $\varepsilon_{q}$ (solid red circles) and damping factor $\gamma_{q}$ (open red circles) versus projected in-plane momentum transfer $q_{/ /}$along high-symmetry directions at $20 \mathrm{~K}$. The dashed curve is linear spin wave dispersion for a two-dimensional antiferromagnetic Heisenberg model fit to the data, with $J_{1}=63.6 \pm 3.3 \mathrm{meV}$ and $J_{2}=-10.3 \pm 2.3 \mathrm{meV}$. The energy resolution of our RIXS measurement is $\sim 37 \mathrm{meV}$, as indicated with the horizontal dashed line. Error bars of $\varepsilon_{q}$ were estimated by combining the uncertainty of zero-energy-loss position, high-energy background, and the standard deviation of the fits. Error bars of $\gamma_{q}$ were estimated by combining the standard deviation of the fits and the uncertainty of high-energy background. 
A

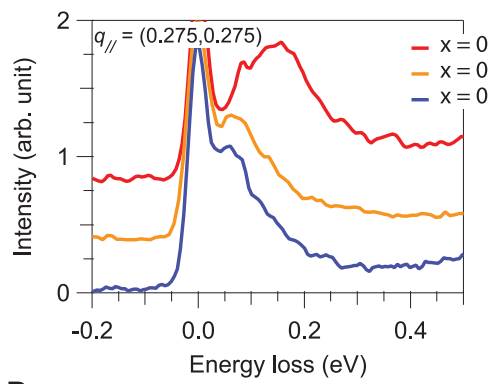

B

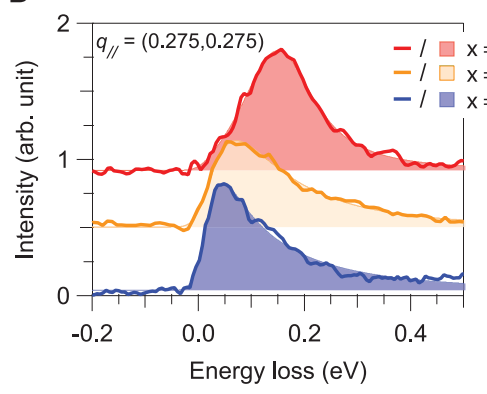

C

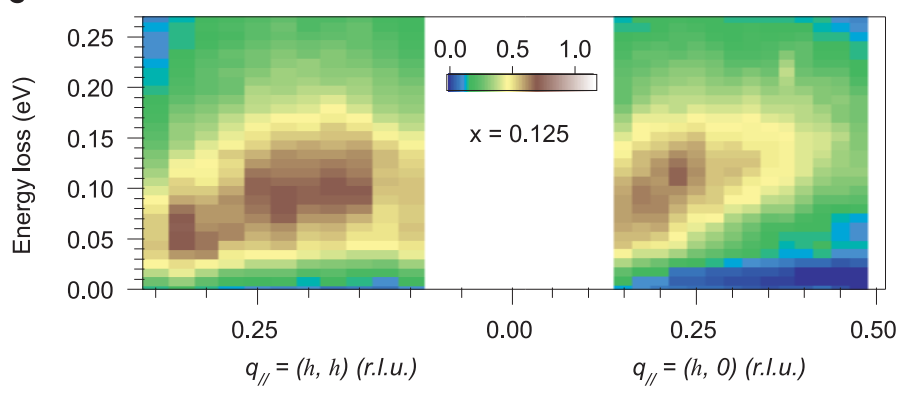

Fig. 4. RIXS spectra of $\mathbf{N d}_{1-x} \mathbf{S r}_{x} \mathbf{N i O}_{2}$. (A) Raw spectra at representative momentum positions for $x=0,0.125$, and 0.225 at $20 \mathrm{~K}$. (B) Magnetic spectra obtained by subtracting the elastic line, phonon, and background from the raw data shown in (A). The shaded areas indicate the associated DHO fitting. (C) Magnetic spectra map of $\mathrm{Nd}_{1-x} \mathrm{Sr}_{x} \mathrm{NiO}_{2}(x=0.125)$ along $h$ and $h$ directions at 20 K. (D to F) Summary of (D) spectral weight, (E) mode energy $\varepsilon_{q}$, and (F)
D

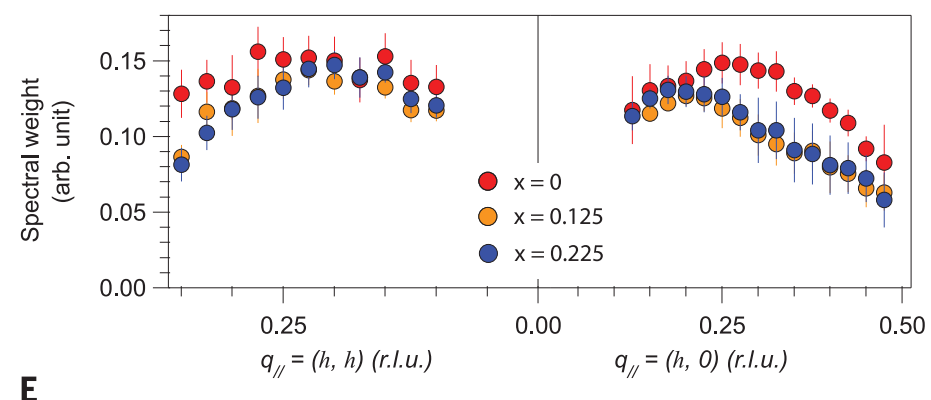

E

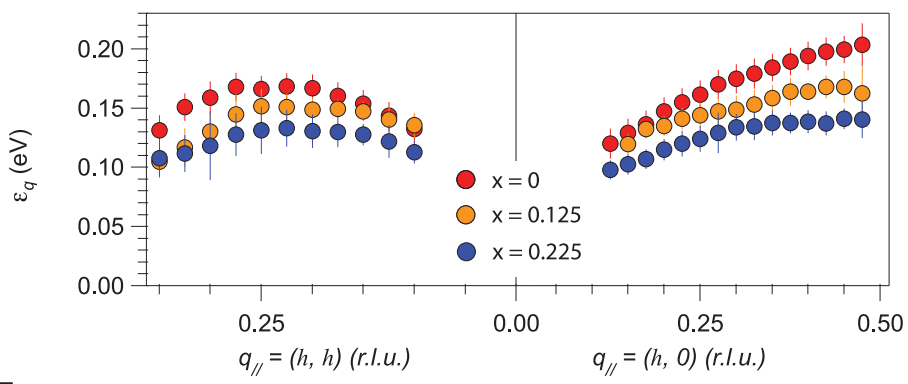

$\mathbf{F}$

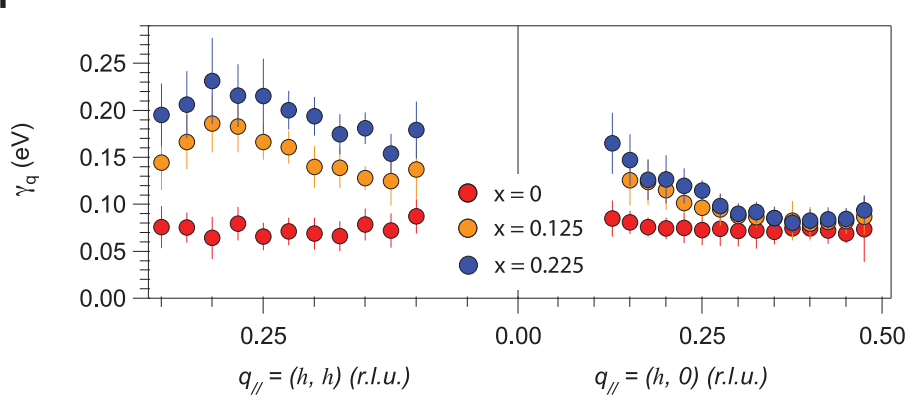

damping factor $\gamma_{q}$ for the three doping concentrations. For $x=0.125$ and 0.225 , error bars of spectral weight and $\varepsilon_{q}$ were estimated by changing the phonon intensity by $\pm 30 \%$, whereas those of $\gamma_{q}$ are the estimate of the standard deviation to the fit and the uncertainty of the high-energy background. For $x=0$, error bars of $\varepsilon_{q}$ and $\gamma_{q}$ are the same as those defined in Fig. 3, and error bars of the spectral weight were derived from the fittings. where $S_{i}, S_{j}$, and $S_{i^{\prime}}$ denote Heisenberg spins at site $i$, nearest-neighbor sites $j$, and the nextnearest-neighbor sites $i^{\prime}$, respectively (27). We found a substantial nearest-neighbor coupling $J_{1}=63.6 \pm 3.3 \mathrm{meV}$ and a sizable next-nearestneighbor coupling $J_{2}=-10.3 \pm 2.3 \mathrm{meV}$.

The magnon bandwidth $(\sim 0.2 \mathrm{eV})$, which measures the strength of the exchange interaction, is quite comparable with that found in the parent cuprates ( 0.3 to $0.4 \mathrm{eV})$ and notably higher than other nickelates $(24,25)$. These results provide clarity to the debate in the theoretical literature on the strength of the exchange coupling (11-17). The theories, which predict a similar value of $J_{1}$, suggest that the effective onsite Coulomb interaction $U$ falls in the range of 5 to $6 \mathrm{eV}$ (15-17). However, we caution that the $U$ value needs to be further refined with experimental values of electron dynamics parameters, which are not yet available. In addition, the magnitude of the next-nearest-neighbor coupling $J_{2}$ is intriguing. In the sense of the Zaanen-Sawatzky-Allen classification, one expects the spin exchange interaction to be rather short ranged because the nickelates are not charge-transfer compounds (10), in which the oxygen ligands serve as a major pathway for the super-exchange interaction (30). Thus, we speculate that this indicates a possible long-range RKKY metallic exchange mediated by the Nd $5 d$ pockets. Although the negative $J_{2}$ (unfrustrated) should stabilize conventional Néel order, neutron powder diffraction studies indicated a lack of long-range magnetic order, despite development of magnetic correlations at low temperatures (31). However, the results of an absent magnetic order may be obscured by the quality of these metastable bulk materials, which is difficult to control. In addition, the heteroepitaxy stabilization by the $\mathrm{SrTiO}_{3}$ substrate could also play a role in sculpting the magnetism in the film, such as through a $\sim 0.4 \%$ compressive strain that is absent in the bulk. Our observations confirm the existence of AFM correlations but do not provide a definitive answer about the existence of long-range AFM order. A direct measurement of the magnetic spectrum at the putative AFM-ordering wave vector is needed, which is unfortunately inaccessible owing to insufficient momentum of the Ni $L$-edge photons. Further investigations that use different experimental probes on both film and bulk compounds are necessary to gain additional insight. 
The other notable behavior revealed by the fits is in the spin wave damping factor $\gamma_{q}$ (Fig. $3)$. The width of the magnetic peaks $\left(\sim 2 \gamma_{q}\right.$ of $\sim 130 \mathrm{meV}$ ) exceeds the value of our instrument resolution $(\sim 37 \mathrm{meV})$. This width is independent of the mode energy and momentum. The fingerprint of itinerant magnetism would be a damping that increases sharply as it moves away from the magnetic ordering wave vector [presumably at $(0.5,0.5)$ ], and thanks to spinwave interactions, the damping should be maximal at the magnetic zone boundary. The rather constant $\gamma_{q}$ suggests that the magnons are instead coupled to a "heat bath," capable of dissipating only small momenta and energy. This may be consistent with the metallic Nd pockets and their small Fermi surfaces, as determined from either tight binding models $(10,15)$ or larger experimental estimates (5). Incomplete chemical reduction and disorder that may suppress long-range antiferromagnetism also can play a role in damping these excitations.

We next discuss the doping evolution of the magnetic excitations in $\mathrm{Nd}_{1-x} \mathrm{Sr}_{x} \mathrm{NiO}_{2}$ from $x=$ 0 to 0.225 , across the superconducting phase boundary (32). As shown in Fig. 4A, the magnetic excitations appear to soften with increasing doping concentration and overlap substantially with phonon excitations toward small momenta (fig. S6A). For those momentum positions, we assume the phonons to be doping independent and deduce magnetic spectra from our fitting analysis as shown in Fig. 4B (27), which contains information about the imaginary part of the dynamical spin susceptibility. As a function of momentum, the magnetic spectra are less dispersive (Fig. 4C and fig. S6B) than those in $\mathrm{NdNiO}_{2}$. The magnetic spectral weight (Fig. 4D) decreases gently with doping, which is consistent with spin dilution as expected in a doped Mott insulator because some spins are replaced by holes. Consequently, the magnetic modes should soften, and the reduced lifetimes would indicate overdamped, relaxational dynamics. To extract the mode energy $\varepsilon_{q}$ and damping $\gamma_{q}$, we fit the data to the DHO function (Eq. 1) (27). The superconducting compound acts as a doped Mott insulator, with $\varepsilon_{q}$ mildly softened compared with the magnons in the parent compound with a similar dispersion (Fig. 4E). The most substantial change is in the $\gamma_{q}$ along the $h h$ direction, which increases greatly compared with the parent nickelate (Fig. 4F), causing substantial asymmetry in the magnetic spectrum (Fig. 4B). Because $\gamma_{q} \gtrsim \varepsilon_{q}$, these high-energy spin excitations in the superconductor are on the verge of becoming overdamped. The DHO fitting is consistent with a model-free spectral moment analysis, validating that our $\mathrm{DHO}$ analysis has captured the essential information of the magnetic spectrum (fig. S7) (27).
Compared with cuprates, which exhibit similar overdamped magnetic modes upon doping, the infinite-layer nickelates show subtle but important differences in the evolution of spectral weight and mode energies. In the cuprates, the spectral weight is found to be essentially unchanged, and the mode energy increases upon substantial doping (22). These effects seem, at first sight, counterintuitive but are rooted in the subtle interplay between magnetic interaction and longer-range hole dynamics, including the next-nearest neighbor hopping and coherent hopping involving multiple sites, such as the three-site exchange interaction (33, 34). The doped nickelates exhibit mild softening and loss of spectral weight that conforms more closely to expectations of the simple $t-J$ model (35). As charge-transfer insulators, the doped holes in cuprates reside mostly on oxygen, implying relatively extended holewave functions, which promote the nonlocal interactions that give rise to the interference effects. The nickelates appear to be more Mott-Hubbard-like (10, 15), implying that hole-wave functions have more localized Ni $d$-like character, leading to a suppression of nonlocal interactions. With the added complexity caused by the presence of the Nd metallic pockets, the upcoming challenge is to find out how these microscopic differences affect collective phenomena in Mott systems. This should give further insight into the relationship between the microscopic ingredients determined with chemistry and the mechanism of superconductivity in these many-bodyentangled quantum materials.

Note added in proof: During the review process, we became aware of RIXS results on triple-layer nickelates, finding similar values of $J$ as in our study (36).

\section{REFERENCES AND NOTES}

1. P. W. Anderson, The Theory of Superconductivity in the High-TC Cuprates (Princeton Univ. Press, 1997).

2. P. Phillips, Ann. Phys. 321, 1634-1650 (2006).

3. J. Berges, Nature 569, 339-340 (2019).

4. J. Zaanen, SciPost Phys 6, 061 (2019).

5. D. Li et al., Nature 572, 624-627 (2019)

6. S. Zeng et al., Phys. Rev. Lett. 125, 147003 (2020).

7. V. I. Anisimov, D. Bukhvalov, T. M. Rice, Phys. Rev. B 59 , 7901-7906 (1999)

8. K. W. Lee, W. E. Pickett, Phys. Rev. B 70, 165109 (2004)

9. A. S. Botana, M. R. Norman, Phys. Rev. X 10, 011024 (2020)

10. M. Hepting et al., Nat. Mater. 19, 381-385 (2020).

11. M. Jiang, M. Berciu, G. A. Sawatzky, Phys. Rev. Lett. 124, 207004 (2020).

12. G.-M. Zhang, Y. Yang, F.-C. Zhang, Phys. Rev. B 101, 020501 (2020).

13. L.-H. Hu, C. Wu, Phys. Rev. Res. 1, 032046 (2019).

14. Z. Liu, Z. Ren, W. Zhu, Z. Wang, J. Yang, npj Quantum Mater. 5 , $31(2020)$
15. E. Been et al., Phys. Rev. X 11, 011050 (2021)

16. V. M. Katukuri, N. A. Bogdanov, O. Weser, J. van den Brink, A. Alavi, Phys. Rev. B 102, 241112 (2020).

17. R. Zhang et al., arXiv:2009.05816 [cond-mat.str-el] (2020)

18. K. Lee et al., APL Mater. 8, 041107 (2020)

19. B. H. Goodge et al., Proc. Natl. Acad. Sci. U.S.A. 118 e2007683118 (2021)

20. L. Braicovich et al., Phys. Rev. Lett. 102, 167401 (2009).

21. M. Le Tacon et al., Nat. Phys. 7, 725-730 (2011)

22. Y. Y. Peng et al., Phys. Rev. B 98, 144507 (2018)

23. K. J. Zhou et al., Nat. Commun. 4, 1470 (2013)

24. G. Fabbris et al., Phys. Rev. Lett. 118, 156402 (2017).

25. Y. Lu et al., Phys. Rev. X 8, 031014 (2018).

26. J. Kim et al., Phys. Rev. Lett. 108, 177003 (2012).

27. Materials and methods are available as supplementary materials.

28. Y. Y. Peng et al., Nat. Phys. 13, 1201-1206 (2017).

29. R. Coldea et al., Phys. Rev. Lett. 86, 5377-5380 (2001).

30. Y. Wang, E. W. Huang, B. Moritz, T. P. Devereaux, Phys. Rev. Lett. 120,246401 (2018)

31. M. A. Hayward, M. J. Rosseinsky, Solid State Sci. 5, 839-850 (2003).

32. D. Li et al., Phys. Rev. Lett. 125, 027001 (2020).

33. C. J. Jia et al., Nat. Commun. 5, 3314 (2014).

34. J. Bała, A. M. Oleś, J. Zaanen, Phys. Rev. B 52, 4597-4606 (1995)

35. P. A. Lee, N. Nagaosa, X.-G. Wen, Rev. Mod. Phys. 78, 17-85 (2006).

36. J. Q. Lin et al., Phys. Rev. Lett. 126, 087001 (2021)

37. H. Lu et al., Replication data for "Magnetic excitations in infinite-layer nickelates". Harvard Dataverse, version 2 (2021)

\section{ACKNOWLEDGMENTS}

Funding: This work is supported by the U.S. Department of Energy (DOE), Office of Science, Basic Energy Sciences, Materials Sciences and Engineering Division, under contract DE-AC02-76SF00515. We acknowledge the Gordon and Betty Moore Foundation's Emergent Phenomena in Quantum Systems Initiative through grant GBMF9072 for synthesis equipment. We acknowledge Diamond Light Source for providing the beam time at the I21-RIXS beamline under proposal NT25165. Author contributions: W.S.L. and K.-J.Z. conceived the research and designed the experiment. H.L., M.R., A.N., M.G.-F., S.A., K.-J.Z., and W.S.L. conducted the experiment at Diamond Light Source. H.L., M.R., A.N., K.-J.Z. and W.S.L. analyzed the data. M.O., D.F.L., K.L., B.Y.W., and H.Y.H. synthesized and characterized samples for the experiment W.S.L., K.-J.Z., H.L., M.R., A.N., D.F.L., H.Y.H., E.M.B., B.M. Z.X.S., T.P.D., and J.Z. discussed and interpreted the results. H.L. and W.S.L. wrote the manuscript, with input from all authors. Competing interests: The authors declare no competing interests. Data and materials availability: All data presented in this work are available online at Harvard Dataverse (37)

\section{SUPPLEMENTARY MATERIALS}

science.sciencemag.org/content/373/6551/213/suppl/DC1 Materials and Methods

Figs. S1 to S7

References $(38,39)$

10 July 2020; resubmitted 8 September 2020

Accepted 21 May 2021

10.1126/science.abd7726 


\section{Science}

\section{Magnetic excitations in infinite-layer nickelates}

H. LuM. RossiA. NagM. OsadaD. F. LiK. LeeB. Y. WangM. Garcia-FernandezS. AgrestiniZ. X. ShenE. M. BeenB. MoritzT.

P. DevereauxJ. ZaanenH. Y. HwangKe-Jin ZhouW. S. Lee

Science, 373 (6551), • DOI: 10.1126/science.abd7726

\section{Looking for magnetic clues}

Thin films of the neodymium nickelate $\mathrm{NdNiO}$ doped with strontium have recently been found to be superconducting.

This materials class bears structural and electronic similarities to the famed cuprate superconductors, but how far the analogy goes remains unclear. Lu et al. used resonant inelastic x-ray scattering to look for magnetism, which exists in the cuprates, in NdSrNiO films (see the Perspective by Benckiser). The authors observed magnetic modes in the undoped compound that had a doping evolution consistent with the behavior of a doped Mott insulator.

Science, abd7726, this issue p. 213; see also abi6855, p. 157

\section{View the article online}

https://www.science.org/doi/10.1126/science.abd7726

Permissions

https://www.science.org/help/reprints-and-permissions 